IP Periodica Polytechnica Electrical Engineering and Computer Science

60(4), pp. 195-199, 2016

DOI: $10.3311 /$ PPee.9739

Creative Commons Attribution (i)

\title{
Design of LTCC Based 3-D Antenna for Sub-THz Application
}

\author{
Beata Synkiewicz $^{1^{*}}$, Jan Kulawik ${ }^{1}$, Yevhen Yashchyshyn ${ }^{2}$, \\ Przemysław Piasecki \\ Received 21 July 2016; accepted after revision 18 August 2016
}

RESEARCH ARTICLE

\begin{abstract}
In the paper, selected aspects of LTCC technology for fabrication of passive elements for microwave application are presented. The research effect is based on the example of experimental 3-D antenna performed for a sub-THz scanner. For the described methods of antennas performance the achieved printing quality have been determined basing on observations using a digital microscope Hirox KH7700 and measurements of geometrical dimensions. The impact of geometrical structure dimensions and printed pattern resolution on its working abilities is also discussed. The main emphasis of the research work is on high resolution metallization obtainment by modification of screen printing parameters and conditions (the angle and pressure of squeegee, the mesh size), laser ablation and co-firing profile.
\end{abstract}

\section{Keywords}

LTCC technology, microwaves, screen printing, laser ablation, ceramics, high resolution patterns

\footnotetext{
${ }^{1}$ Institute of Electron Technology, Krakow Division,

ul. Zabłocie 39, 30-701 Kraków, Poland

${ }^{2}$ Warsaw University of Technology,

Institute of Radioelectronics and Multimedia Technology

ul. Nowowiejska 15/19, 00-665 Warsaw, Poland

"Corresponding author, e-mail: synkiewicz@ite.waw.pl
}

\section{Introduction}

The rapid growth of the wireless communication industry as the current trends towards miniaturization has stimulated development of new materials and devices operating properly at high frequencies. The fabricated materials can be useful for substrates of microwave devices and antennas. Dielectric properties of the passive elements in devices working at high frequencies are determined by a lot of factors (for example printed patterns resolution, obtainment of accurate 3-D dimensions of the substrate, smooth surface of metallization, etc.). In face of demand for new applicable elements working at microwaves it was decided to create a passive element (antenna) basing on LTCC technology. Low temperature co-fired ceramic (LTCC) technology is suitable for creating multilayer (3D) structures, integrating conductive paths, passive inductors and resistors and resonance cavities. In the fabrication of LTCC structures, green ceramic tapes are mechanically processed in order to form holes and vias, which can be performed by laser processing. Another application of a laser in LTCC technology is ablation of printed metallic layer for desired conductor pattern [1-3] obtainment.

The most important advantage of the LTCC is possibility to create multifunctional structures thanks to the multilayer integration of green tape with different microstructure, composition, and specific electrical or mechanical properties. Our previous research has been focused on attaining good dielectric characteristics of LTCC structures at microwave frequencies [4, 5]. The quality of patterns screen printed on LTCC substrates is dependent on several factors at each stage of technological path displayed schematically in Fig. 1. To get the best resolution of screen printed patterns it is needed to obtain smooth and nonporous surface of the ceramic structure (eliminating absorption of humidity and solvents) Second substantial aspect is optimization of screen printing technology (both screen preparation and printing conditions). In case of insufficient precision relatively to the geometrical regime for micropatterns, local laser ablation of the metallic layer is desired. 


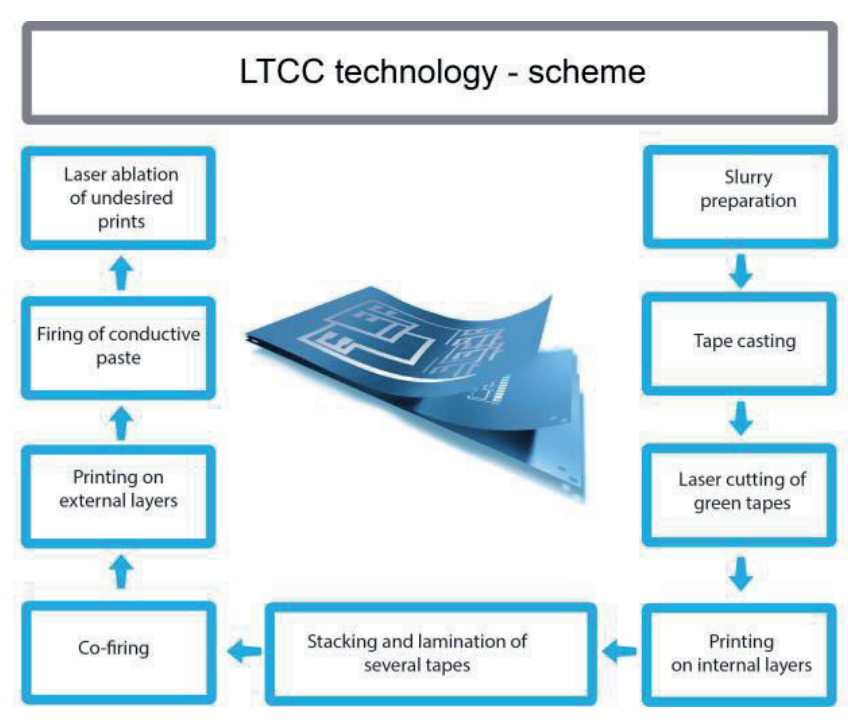

Fig. 1 Scheme of LTCC technological path

\subsection{LTCC technology advantages}

The LTCC technology is designed to produce, among others, complex multifunctional packages for microelectronic circuits and passive components, such as resistors, capacitors and coils. The main recipients of LTCC in the 90 's were such industries as military, medical, automotive and electronic for telecommunication. In the recent years, the latter domain has dominated the LTCC market and, at present, the major actors in the manufacturing business, such as United States and Japan, are working on miniaturisation of electronics for telecommunication (cellular telephony, local Bluetooth network, GPS or global satellite Internet) with this multi- layered technology [6]. The key advantages and possibilities of the LTCC technology are listed below.

- Due to its resistance to mechanical effects, temperature variations and humidity, it is possible to manufacture a cooling and heating installation with a system of heaters (using the possibility to integrate heaters and cooling channels within the structure) and temperature sensors.

- All passive components (conductive paths, inductive components, resistors, capacitors etc.) can be made in a single process with a package, without the need to use alternative methods (e.g. SMD) which slows the production down.

- It is possible to integrate, within the system, micromechanical MEMS devices, such as sensors, levers, actuators, etc.

- The structures may exhibit various electrical properties by manipulating with the composition of ceramic tape and pastes used for printing or because of metallisation of interlayer connections (in addition to that, LTCC integrates well with semiconductors).

- The LTCC structure can have any shape, air or liquid ducts, any notches, holes and connections between the layers made by vias (with a diameter from 100 to
$200 \mu \mathrm{m})$ filling with conductive paste, thus ensuring flexibility in designing spatial structures (also the passive components, including coils, filters and antennas) [7]. The requirements for ideal insulating substrates are primarily: high resistivity, low dielectric constant, low dielectric loss (dissipation factor), thermal expansion coefficient adjusted to semiconductor structures (for silicon it is $3 \cdot 10-6 /{ }^{\circ} \mathrm{C}$, for $\mathrm{GaAs}-6 \cdot 10-6 /{ }^{\circ} \mathrm{C}$ ), high mechanical strength, good chemical and thermal stability, suitability for co-firing with conductive layers.

LTCC is a process of multilayer integration of sheets with defined and limited thickness. Many materials (so called green tapes) are commercially available for this purpose, however their thickness ranges are from several dozens up to several hundreds of micrometers (medium and most popular size is about 100 micrometers) which compared with wavelength above $100 \mathrm{GHz}$ provides a very poor resolution. Also if some roughness or deformations between each of layers will occur, then it gives additional errors in electrical parameters of designed structure. However, the conductive layer which specifies the device structure is one of the important constituent elements of mm-wave devices. Application of conductive paste onto a green tape by screen printing and in the next step co-firing of the structure or the change of this order - first co-firing and in the next step printing can lead to most desired effects. In the mm-wave range there are two aspects associated with conductive structure: the effect of decreased conductivity (conductivity is lower in microwave frequencies [8]) and precision mapping structure. It is necessary to use materials with very high conductivity comparable to $\mathrm{Cu}$, $\mathrm{Au}, \mathrm{Ag}$ or metals alloyed with them. Roughness of the substrate is crucial because of a depth skin effect, (e.g. for Ag at $100 \mathrm{GHz}$ is about $0.2 \mu \mathrm{m}$ ). Irregular metallization. can be the caused by improper screen printing manufacture technology. Conductivity and roughness of metal structures have a significant influence on the whole device parameters including efficiency. Furthermore, precisely manufacturing of conductive structure of devices, e.g. lines, slots, couplers or filters is demanded. Every inaccuracy of conductive structure's production may affect the operating frequency band of devices or the operation of the devices at all. In addition to the mentioned aspects reducing the inaccuracies of the whole 3D LTCC structure as compared to the design project is very much expected.

\section{Results}

\subsection{3-D antennas design and construction}

The effects of research are presented basing on the example of high gain 3D antennas for sub-THz frequency performed using LTCC technology. Considering the manufactured antenna as an example (Fig. 2) of device which weakly depends on the transverse dimensions, changing of W (Fig. 2) width of radiation structure of $125 \mu \mathrm{m}$ (which is around $6 \%$ of wavelength) 
alters around $2 \%$ the antenna frequency resonance, as can be seen in Fig. 3.

The $2 \%$ shifting is not a big value, but it is comparable to the operating bandwidth of antenna.

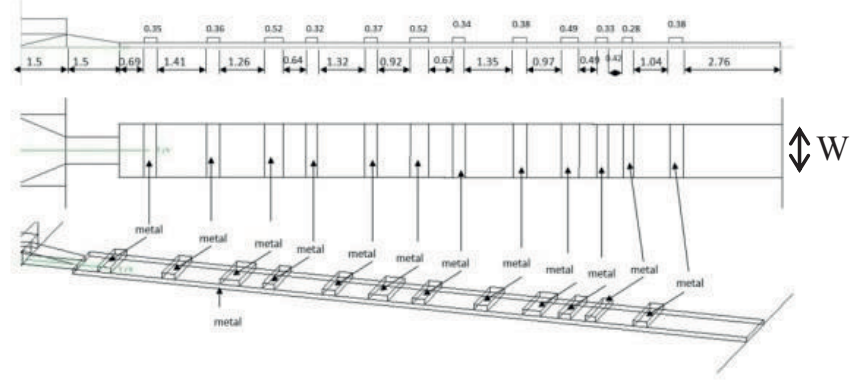

Fig. 2 Design of integrated antenna module for realization and integration in LTCC technology

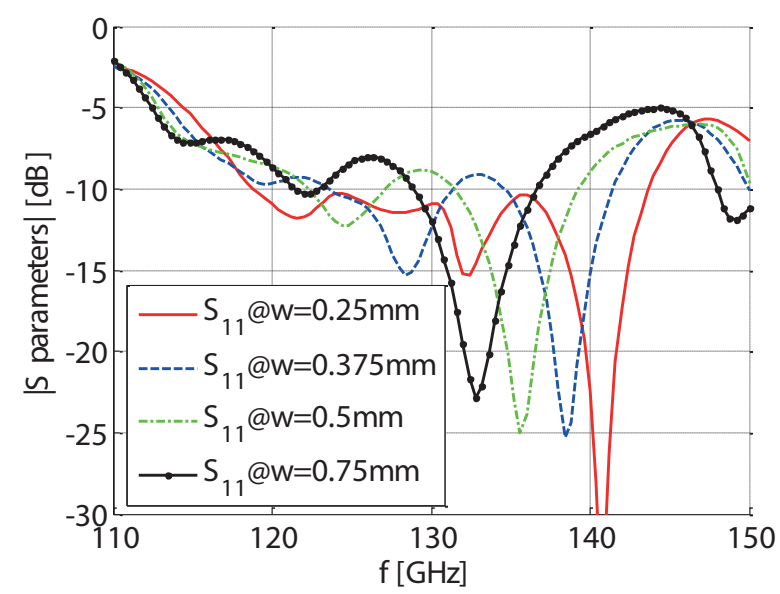

Fig. 3 Simulated results of changing $\mathrm{W}$ dimension of the mm-wave antenna

This case illustrates that dimensions keeping, especially in mm-wave range, is essential during a manufacture process. The accuracy of LTCC technology depends on many things that are done throughout the whole production process. For example, in order to create a proper metallization pattern, screen printing or photolithography technics can be used, but in both cases the final results have to be more or less similar to these simulated in an electromagnetic simulator. What is more, some technological limitations of LTCC technology cannot be avoided, like limitation of vias diameter or minimal distance of vias to the edge of signal lines or the end of designed structure. For these reasons, during designing process a lot of simulations have to be done to be sure that both simulated and manufactured structures will give-good electrical results consistent with themselves.

3D antennas were designed and screen printed using a silver paste (ESL 9916) on ceramic substrates with integrated small ceramic bars (width 280-520 $\mu \mathrm{m}$ ). To compare the effects caused by different technological solutions at the angle of printed pattern resolution, experimental prints on the LTCC substrate were performed using three process modifications:
- Printing of the pattern on LTCC green tape (DP 951), then laser treatment, lamination and co-firing of 3D structure (Table 1) [9].

Table 1 Profile of co-firing of multilayer green-tape

\begin{tabular}{lll}
\hline Temperature $\left[{ }^{\circ} \mathrm{C}\right]$ & Heating time $[\mathrm{h}]$ & Holding time $[\mathrm{h}]$ \\
\hline 100 & $0: 30$ & $0: 30$ \\
400 & $3: 00$ & $2: 00$ \\
650 & $2: 00$ & $1: 00$ \\
850 & $1: 30$ & $0: 30$ \\
400 & $5: 00$ & $0: 00$ \\
20 & cooling with furnace & - \\
\hline
\end{tabular}

Laser embossing of external green ceramic sheets, lamination and co-firing of multilayer 3D structure and in the next step printing of conductive paths on its surface (Fig. 4).

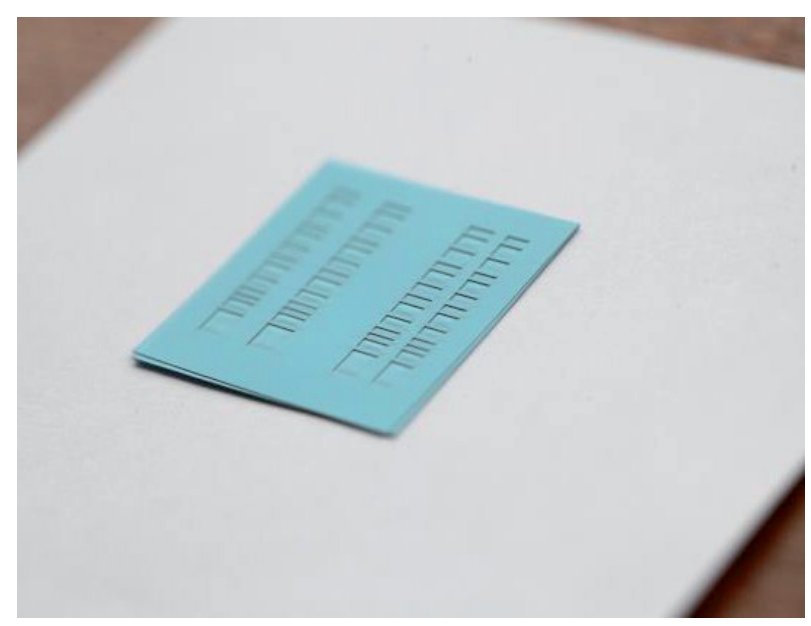

Fig. 4 Multilayer laminated green-tape structure after first laser ablation.

Printing of conductive paths on 3D co-fired substrate and their laser ablation for obtainment of a high resolution pattern (Fig. 5).

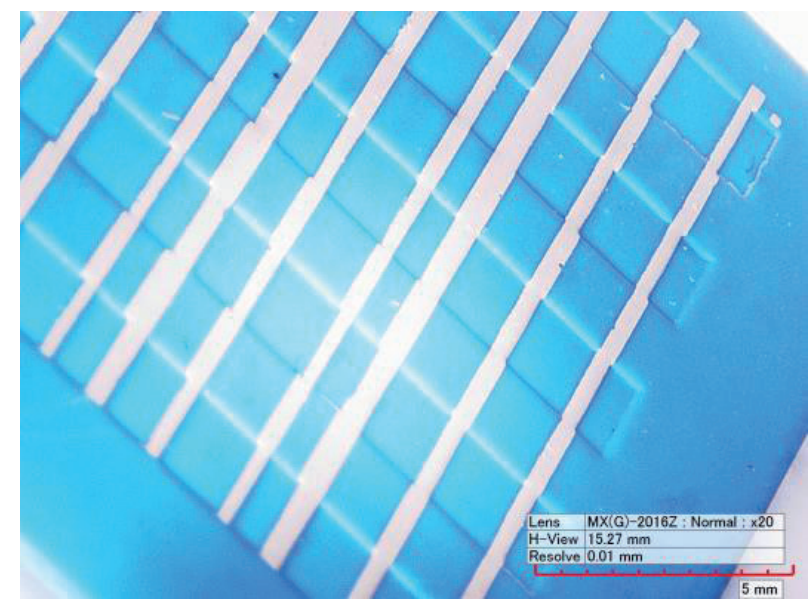

Fig. 5 Printed co-fired 3-D LTCC structure - substrate for antenna. 
For the described methods, the achieved pattern resolution and quality have been determined basing on observations using a digital microscope Hirox KH7700 and measurements of geometrical dimensions. The results indicate that the designed mm-wave structure is sensitive for variation of the radiated structure width. This sometimes can be desired feature or unwanted drawback, depending on the application.

\subsection{Screen printing limitations}

Screen printiing is one of the most widely known deposition methods in microelectronics, fully compatible with thick-film technology [10]. In case of LTCC this technique is based on the transfer of specialized (resistor, dielectric, piezoelectric, glass) pastes onto the surface of green (not-fired) ceramic substrate (except in the masked areas impermeable to the paste). In the next step the paste is fired with the substrate during cofiring process and the pattern becomes fixed. Unfortunately, the structure of the screen is one of a lot of factors determining resolution and quality of the printed pattern. The most important is diameter of the mesh opening. The most accurate patterns are usually printed through the screens with density about 600 mesh and higher (that gives about 30-40 $\mu \mathrm{m}$ of screen hole size, and such the resolution). Another aspect influencing printing quality is the amount of the paste going through the mesh during print. A squeegee is moved through the surface of the screen to fill the open mesh apertures with paste. Then a reverse stroke enables the screen to touch the substrate instantaneously along a line of contact. During this contact the paste is moved through the screen. Quality of this process is dependent on temperature of the room, rheology of paste and thickness of the light-sensitive emulsion (impermeable substance). At higher temperatures, the paste becomes more fluid and that creates a danger of spillage on the surface in undesired areas of the substrate and creation of short circuits or irregularities of the pattern. If the splinters of the paste appeared several times deeper, it would introduce modulation of electromagnetic waves and as a result change the characteristics of radiation and could have an impact on the standing wave ratio (SWR) in the structure.

\subsection{Laser ablation}

In case of unwanted irregularities of print, we attempt to correct errors using a pulsed Nd:YAG laser CNC machine (355 $\mathrm{nm}$ ). Output power of the laser was varied by setting the attenuation percentage. Laser source works at a constant power (maximum $10 \mathrm{~W}$ ), while laser beam is attenuated by passing through a set of polarizing filters. For the investigation of optimal laser ablation parameters, output power was varied between $12.5 \%$ and $100 \%$. Also, laser pulse frequency, i.e. number of pulses generated per second, was varried (four steps from $2.5 \mathrm{kHz}$ up to $17.5 \mathrm{kHz}$ ). Machining speed, i.e. speed of table movement during exposure on laser beam on the processed substrate, was varied from $2.5 \mathrm{~mm} / \mathrm{s}$ to $20 \mathrm{~mm} / \mathrm{s}$.
The effect of laser ablation (depth and precission of cut line) was observed and measured basing on Hirox KH 7700 microscopic observation. The use of this method was described in our previous work [11]. The dependence of depth of laser ablation on the applied parameters of the laser operation is shown in Fig. 6. Basing on the observations and results of measurements we concluded, that it is possible to remove unwanted printed paste after co-firing of the structure with nondestructive influence on the substrate surface.

Conductive layer removal without substrate destruction was attainable for the chosen parameters of the laser: power of $37.5 \%$ (maximum power $-10 \mathrm{~W}$ ), frequency of $2.5 \mathrm{kHz}$, speed of table movement of $17.5 \mathrm{~mm} / \mathrm{s}$. As the result, $56 \mu \mathrm{m}$ depth of laser cutting and total removal of metallization were obtained. The width of ablated area reached $47 \mu \mathrm{m}$ giving the opportunity to increase the resolution of the pattern (Fig. 6).

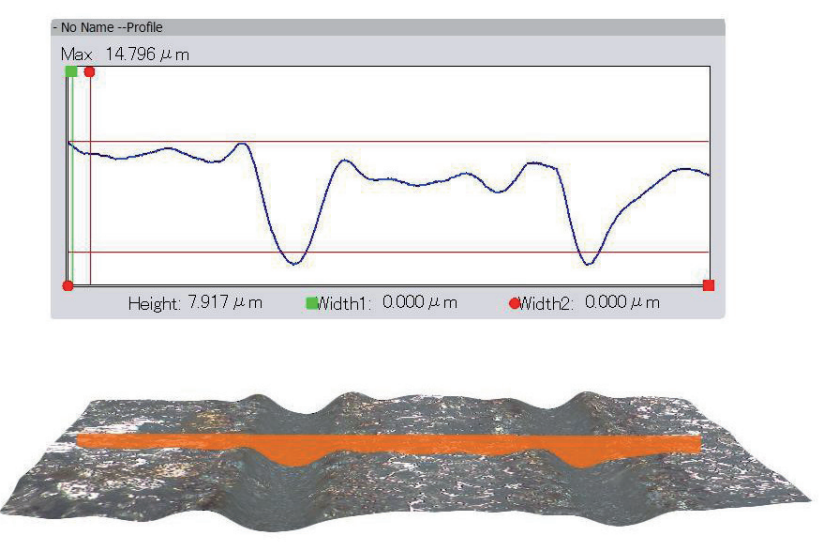

Fig. 6 The effect of laser ablation on LTCC substrate (3-D profile, Hirox KH7700)

\section{Discussions}

Screen-printing of the pattern before lamination and co- firing, led to cracking of the structure due to different shrinkage of the LTCC green tape and conductive paste and thermal expansion mismatch (Fig. 7).

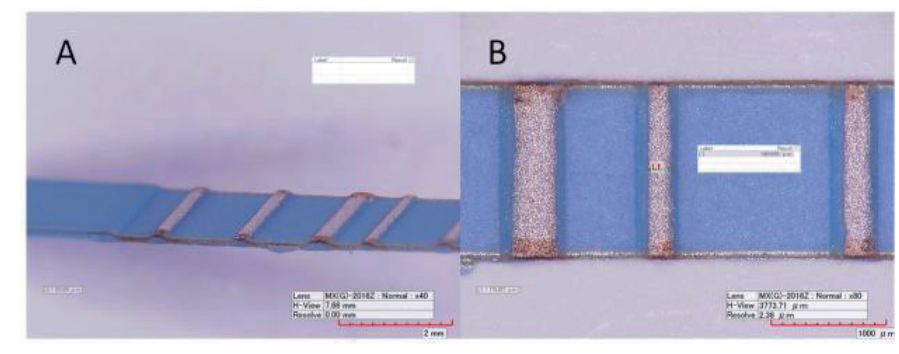

Fig. 7 Antenna printed after laser processing and co-firing.

Width of the conductive paths is about $80-180 \mu \mathrm{m}$. Printing after co-firing prevented such deformation of the substrate. In the second method - the use of screen polyester mesh 380 and higher and the angle of the squeegee about $35^{\circ}$, gave opportunity to reach straight lines (width of $47-70 \mu \mathrm{m}$ ) of high 
resolution after co-firing. Samples of the obtained structures are presented in Fig. 7A and Fig. 7B. Geometric measurements revealed that optimization of the squeegee pressure is required to minimize the observed distortion of the pattern and its improper fitting to the substrate (up to $10 \%$ deviation). After optical inspection of the created 3-D antenna, it was found that, after minor modifications enabling connection to the waveguide and minimization of shape distortion, the device is useful to work at $100 \mathrm{GHz}$ wavelength.

\section{Conclusions}

It is possible to perform three-dimensional sub- $\mathrm{THz}$ antenna based on LTCC technology.

Small remnants of metallization as single defects should have no effect on the antenna performance and can be removed by laser ablation using $2.5 \mathrm{kHz}$ frequency, power of $3.7 \mathrm{~W}$ and speed of table movement of $17.5 \mathrm{~mm} / \mathrm{s}$.

At a later stage of the research it is planned to simplify the transition from the waveguide WR- 8 to the radiating structure, which will be an integral part of the antenna and to minimize the deformation caused by the contraction of three-dimensional space by slowing the firing process.

\section{Acknowledgement}

This study was partially financed by National Centre for Research and Development, Poland as PBS Project LTCC No. 244389 and as ITE Scientific Research PhD Project 53.06.008.

\section{References}

[1] Zaraska, K., Gaudyn, J., Bieńkowski, A., Czerwiński, A., Płuska, M. "Surface Properties of Laser-Etched LTCC Ceramic." In: Proceedings of the 44th International Symposium on Microelectronics, Long Beach, USA, Oct. 9-13, 2011, pp. 735-739.

[2] Shafique, M. F., Saeed, K., Steenson, D. P., Robertson, I. D. "Laser Prototyping of Microwave Circuits in LTCC Technology." IEEE Transactions on Microwave Theory and Techniques. 57(12), pp. 3254-3261. 2009. DOI: 10.1109/TMTT.2009.2033846

[3] Bieńkowski, A., Gaudyn, J., Zaraska, K., Rydosz, A., Maziarz, W., Malecha, K. "Laser Micromachined LTCC Gas Sensors." Elektronika. 52(3), pp. 90-92, 2011.

[4] Kulawik, J., Szwagierczak, D., Synkiewicz, B. "Fabrication of CaCu3Ti4O12 Multilayer Capacitors, Their Microstructure and Dielectric Properties." Ferroelectrics. 447(1), pp. 19-32.

DOI: 10.1080/00150193.2013.821385

[5] Kulawik, J., Szwagierczak, D., Synkiewicz, B. "Multilayer ceramic capacitors with CaCu3Ti4O12 dielectric." In: Proceedings of ISAFECAPD-PFM 2012, Aveiro, July 9-13, 2012, pp. 1-4. DOI: 10.1109/ISAF.2012.6297757

[6] Golonka, L. "Zastosowanie ceramiki LTCC $w$ mikroelektronice." (The application of LTCC in microelectronics.) Oficyna Wydawnicza Politechniki Wrocławskiej, 2001.

[7] Dziedzic, A. "Electrical and structural investigations in reliability characterisation of modern passives and passive integrated components." $\mathrm{Mi}$ croelectronics Reliability. 42(4-5), pp. 709-719. 2002. DOI: 10.1016/S0026-2714(02)00044-6

[8] Samanta, K. K., Robertson, I. D. "Characterisation of TFMS and CPW Lines and Interconnections up to $100 \mathrm{GHz}$ in Multilayer Photoimageable Thick Film Technology." In: 2006 European Microwave Conference, Manchester, Sept. 10-15, 2006, pp. 376-379.

DOI: 10.1109/EUMC.2006.281352

[9] DuPont USA. [Online]. Available from: http://www.dupont.com/

[10] Jurków, D., Maeder, T., Dąbrowski, A., Santo Zarnik, M., Belavič, D., Bartsch, H., Müller, J. "Overview on low temperature co-fired ceramic sensors." Sensors and Actuators A: Physical. 233, pp. 125-146. 2015. DOI: 10.1016/j.sna.2015.05.023

[11] Zaraska, K., Machnik, M., Bieńkowski, A., Synkiewicz, B. "Depth of Laser Etching in Green State LTCC." In: Proceedings of the IMAPS/ACerS 8th International CICMT Conference and Exhibition, Erfurt, April 16-19, 2012, pp. 136-141. 